\title{
Percepção do agente comunitário sobre o Programa Família Saudável*
}

\author{
Perception of the community health agent about the Healthy Family Program \\ Percepción del agente comunitario sobre el Programa Familia Saludable
}

\author{
Stella Maris Hildebrand', Helena Eri Shimizu' \\ 'Universidade de Brasília, Faculdade de Ciências da Saúde, Departamento de Enfermagem. Brasília, DF
}

Submissão: $26 / 10 / 2007$

Aprovação: 14/04/2008

\section{RESUMO}

O objetivo é conhecer a percepção do Agente Comunitário de Saúde sobre o Programa Família Saudável a fim de identificar as potencialidades e fragilidades para mudança do modelo de atenção. Trata-se de um estudo Qualitativo, realizado na unidade de Família Saudável de São Sebastião, DF. A coleta de dados consistiu na observação do processo de trabalho e entrevistas com Quatro Agentes Comunitários de Saúde, analisadas com a técnica do Discurso do Sujeito Coletivo. Os resultados demonstraram Que o objeto de trabalho do PSF são os problemas de saúde encontrados na comunidade, onde são desenvolvidas ações básicas cuja finalidade é descongestionar serviços de saúde. Conclui-se que o Programa Família Saudável caminha em direção a mudanças, sobretudo porQue possibilita a criação de vínculo com a comunidade.

Descritores: Enfermagem em saúde comunitária; Saúde da família; Atenção básica.

\begin{abstract}
This study aims at investigating the perceptions of the Community Health Agent regarding the Healthy Family Program in order to identify strengths and weaknesses, for changing the health care attention model. This is a Qualitative study, carried out in the Healthy Family Unit of São Sebastião, Federal District. Data were collected by observing the process of work and from interviews with four Community Health Agents, analyzed through the technique of the Collective Subject Speech. The results demonstrated that the object of the Healthy Family Program are the community health problems; basic actions are developed, whose objective is to reduce congestion in health services. It was concluded that the Healthy Family Program is moving toward changes, above all because it promotes the creation of liaisons within the community.
\end{abstract}

Descriptors: Community health nursing; Family health; Basic attention.

\section{RESUMEN}

El objetivo es conocer la percepción del Agente Comunitario de Salud sobre el Programa Familia Saludable, con el fin de identificar las potencialidades y fragilidades para cambios en el modelo de atención. Se trata de un estudio cualitativo, realizado en la unidad de Familia Saludable de São Sebastião, Distrito Federal. La obtención de datos consistió en la observación del proceso de trabajo y en entrevistas con cuatro Agentes Comunitarios de Salud, analizadas con la técnica del Discurso del Sujeto Colectivo. Los resultados demostraron Que el objeto de trabajo del Programa Familia Saludable consiste en los problemas de salud de la comunidad. Se desarrollan acciones básicas, cuya finalidad es descongestionar los servicios de salud. Se concluye que el Programa Família Saludable camina en dirección a cambios, sobre todo porque posibilita la creación de vínculos con la comunidad.

Descriptores: Enfermeria en salud comunitaria; Salud de la familia; Atención básica.

*Pesquisa financiada pela FUNPE/UnB 2005 e Conselho Nacional de Desenvolvimento Científico e Tecnológico (CNPQ) - Processo 401950/2005-I.

Correspondência: Stella Maris Hildebrand. Universidade de Brasília. Faculdade de Ciências da Saúde. Campus Universitário Darcy Ribeiro. CEP 70910-900 - Brasília, DF. 


\section{INTRODUÇÃO}

Os Agentes Comunitários de Saúde (ACS) integram a força de trabalho em saúde no Brasil desde as primeiras experiências de ampliação de cobertura dos serviços de atenção básica, tais como Serviço Especial de Saúde Pública (SESP), Fundação Serviço Especial de Saúde Pública (FSESP), Programa de Interiorização de Ações de Saúde e Saneamento (PIASS) e outras experiências estaduais e/ou municipais Que se mostraram capazes de ampliar o cuidado à saúde de populações de risco ${ }^{(1-3)}$.

Em 1991, com a criação do Programa Agente Comunitários de Saúde (PACS), o trabalho do ACS voltou a ganhar nova expressão, sobretudo porQue as suas ações durante a implantação estadual contribuíram com a redução da mortalidade infantil no Ceará no período de 1987 a 1990 e em 1991, no Amazonas trabalhando no combate à cólera surgida no Alto do Solimões ${ }^{(2,3)}$. Posteriormente, em decorrência dos resultados positivos do PACS, foi criado, em 1994, o Programa Saúde da Família (PSF) ${ }^{(4)}$, Que passou a ser considerada estratégia de reordenação do modelo de atenção à saúde do Brasil, para o fortalecimento dos princípios e pressupostos do Sistema Único de Saúde (SUS) ${ }^{(5)}$.

A Estratégia Saúde da Família (ESF) propõe um novo modo de cuidar da saúde, tendo a família como objeto de atenção, considerando-a em seu contexto social, econômico e cultural; estabelece Que uma equipe multiprofissional seja responsável pela saúde da população adstrita nas ações de prevenção da doença, promoção e tratamento da saúde e composta por médico, enfermeiro, auxiliares de enfermagem, ACS e um dentista, a partir de 2000 $(\mathrm{MS})^{(4-6)}$

Da equipe multiprofissional, o trabalho do ACS merece destaQue, pois Ihe são atribuídas funções estratégicas e complexas, sobretudo relacionadas ao contato direto com a comunidade. São funções do ACS: realizar o mapeamento de sua área de atuação, cadastrar e acompanhar as famílias de seu território, identificar situações de risco, desenvolver ações básicas de saúde de prevenção e promoção, promover a educação à saúde e a mobilização comunitária mediante ações de saneamento e melhoria do meio ambiente, incentivar a formação dos Conselhos Locais de Saúde, orientar as famílias para a utilização adequada dos serviços de saúde, informar os demais membros da equipe acerca da dinâmica social da comunidade até participar do processo de programação e planejamento local das ações com vistas à superação dos problemas identificados ${ }^{(5)}$

O ACS representa o maior contingente da força de trabalho na atenção às famílias, atualmente, 204 mil estão em atividades em 5,2 mil municípios, e podem ser encontrados em duas situações distintas em relação à rede do SUS: a) ligados a uma unidade básica de saúde ainda não organizada na lógica da Saúde da Família; e b) ligados a uma unidade básica de Saúde da Família como membro da equipe multiprofissional. Estima-se a cobertura de 58,4\% da população brasileira, o Que corresponde a cerca de 103,5 milhões de pessoas ${ }^{(6)}$.

Ressalta-se, Que o ACS tem preparo profissional peculiar, Que se baseia na sua experiência como morador da comunidade e uma formação técnica relativamente curta com predomínio do enfoque biológico ${ }^{(7)}$. Nesse sentido depreende-se Que a formação de sua identidade é híbrida e polifônica, pois contém o conhecimento científico e do senso comum, Que pode permitir um diálogo profícuo entre os dois saberes ${ }^{(7)}$.

O ACS é considerado o elo entre a equipe de saúde e comunidade, tendo como função precípua transmitir conhecimentos científicos para o universo popular, facilitando o acesso dos usuários aos serviços de saúde ${ }^{(8)}$. Dois atributos sociais básicos do ACS são apontados: identidade com a comunidade e pendor para a ajuda solidária que aliados à sua capacidade de liderança o tornam um mediador entre duas esferas: o Estado e a comunidade ${ }^{(8)}$.

Sob este tema Nogueira et al ${ }^{(8)}$ evidenciaram Que a utilização do ACS pela política social revela duas dimensões contrárias: a "utilitarista" Que usa esse trabalhador como mão de obra de baixo custo e insubstituível pelo seu conhecimento sobre comunidade e as formas como se relaciona com ela; e a "solidarista" Que vê neste profissional o reconhecimento de seu potencial e sua valorização no mercado formal como recompensa e direito.

Verifica-se Que o ACS tem inserção singular na atenção à saúde da família, exerce ações estratégias, portanto têm importantes contribuições no processo de trabalho, principalmente porque agregam no seu trabalho as dimensões: política, técnica, comunicativas nas relações estabelecidas entre serviços de saúdecomunidade $\mathrm{e}^{(1,3,7.8)}$

Há Que se salientar Que são necessárias transformações no processo de trabalho para Que ocorram mudanças no modelo de atenção ${ }^{(9-15)}$, entendido como a maneira de combinar saberes e tecnologias para intervir sobre problemas de saúde e atender as necessidades de saúde individuais e coletivas ${ }^{(9)}$, no que tange a redefinição do objeto, meios e instrumentos e finalidade ${ }^{(9-14)}$, o Que demanda análise dos saberes e tecnologias, das relações estabelecidas entre os sujeitos, profissionais e trabalhadores da saúde, e a população usuária do sistema ${ }^{(10-15)}$.

\section{OBJETIVO}

Analisar as percepções dos ACS sobre o processo de trabalho no PSF a fim de verificar as potencialidades e fragilidades das práticas desenvolvidas para mudanças no modelo de atenção.

\section{PERCURSO METODOLÓGICO}

Trata-se de um estudo Qualitativo. A Unidade Tradicional de Saúde de São Sebastião do Distrito Federal (DF) foi escolhida como local de estudo, por atender os seguintes critérios de inclusão: estar em funcionamento pelo menos há seis meses, ter o maior número de equipes em funcionamento, ter instalação física exclusiva para o programa, ser unidade urbana e possuir equipe completa (médico, enfermeiro, dois auxiliares de enfermagem e Quatro ACS).

A coleta de dados foi realizada em duas etapas, a primeira consistiu na realização de entrevistas com Quatro ACS, utilizandose de um roteiro semi-estruturado contendo Questões sobre o trabalho desenvolvido, as dificuldades e percepções sobre o PSF, Que posteriormente foram transcritas na íntegra. A segunda foi dedicada à observação da atuação do ACS no cotidiano do trabalho no desempenho das ações: visitas domiciliares, palestras educativas, reunião de equipe, relatórios e registros de produção, organização da unidade e apoio às atividades de consulta médica e de enfermagem, durante três semanas, totalizando 40 horas, com registro em diário de campo. Foram treinados Quatro auxiliares de pesquisa para a coleta de dados, sob a supervisão presencial de uma das pesquisadoras no local e semanalmente pelas duas pesquisadoras em reuniões de acompanhamento e orientação.

Para analisar os depoimentos elegeu-se a técnica do Discurso do Sujeito Coletivo - DSC ${ }^{(16,17)}$, pois possibilita a visualização da percepção coletiva na medida em Que permite captar o discurso Que revela o modo como os indivíduos reais e concretos pensam. As etapas de análise foram: leitura flutuante dos depoimentos, destaque das expressões-chave e das idéias centrais (IC), por fim 
a construção do DSC Que é redigido na primeira pessoa do singular e composto pelas ECH Que têm a mesma IC.

Emergiram dos depoimentos Quatro discursos coletivos contendo a percepção dos ACS sobre o objeto de trabalho do PSF (DSC 1), as práticas desenvolvidas pelo PSF (DSC2), a finalidade do PSF, o acesso aos serviços de saúde (DSC3) e a necessidade de continuidade e dificuldades dos do PSF (DCS4)

Os dados obtidos nas entrevistas foram analisados, conjuntamente, com os registros feitos a partir das observações e anotados em diário de campo, com a finalidade de retratar e aprofundar a compreensão das vivências cotidianas do ACS no PSF.

A pesquisa seguiu os procedimentos formais recomendados pela Resolução 196/96 do CNS, foi aprovada pelo Comitê de Ética em Pesquisa da SES/DF e da FS/UnB e os participantes assinaram o Termo de Consentimento Livre e Esclarecido.

\section{RESULTADOS E DISCUSSÃO}

\section{DSC 1: Percepção do ACS sobre o objeto de trabalho do PSF}

O PSF trabalha com os problemas de saúde da comunidade, para trazer um melhor atendimento à comunidade e para aproximar a comunidade da saúde. O ACS encontra na casa das pessoas os problemas e as dificuldades de saúde. O ACS capta os problemas e trás para a unidade pra discutir com os demais profissionais $O$ Que fazer. É um trabalho em equipe, se eu não funcionar o médico não funciona. Se eu não funcionar o enfermeiro não funciona e se o médico não funcionar, a enfermeira e nem eu funcionamos. Que nós somos um elo, somos a continuidade um do outro (DSCI).

O discurso coletivo revela Que o objeto de trabalho do PSF é a saúde da comunidade. Nota-se, porém, Que predomina a percepção de Que a atenção à saúde pauta-se nas ações de identificação e resolução de problemas de saúde de acordo com saberes e fazeres do "modelo clínico hegemônico". Essa influência revela-se na perspectiva de recuperar, restaurar o corpo anátomo-patológico para devolvê-lo a sociedade, à vida e ao trabalho na condição de normalidade produtiva ${ }^{(18)}$.

Entretanto, emerge do discurso uma nova lógica para a apreensão do cuidado à saúde, com o deslocamento do agente do processo de trabalho até as pessoas que residem em suas casas, ruas e microáreas, ou seja, nos espaços de vida dos usuários do serviço.

O DSC I evidencia Que, embora ainda não reconheçam como objeto as "necessidades sociais de saúde"(13), ou seja, para além dos problemas da saúde identificados como os riscos, agravos, doenças e mortes, existem indícios da incorporação dos elementos do conceito ampliado de saúde. Nesse sentido, os dados sugerem um processo de transição nas concepções e práticas pautadas no modelo tradicional da saúde pública ${ }^{(11,19)}$ para àQuele Que visa garantir o conceito ampliado de saúde proposto pelo SUS ${ }^{(12)}$.

Ressalta-se, contudo, Que DSCI não revela a percepção da saúde como direito do cidadão e dever do Estado mediante políticas públicas universais contidas na legislação brasileira ${ }^{(13,20)}$.

O coletivo identificou em sua prática Que se trata de um objeto complexo Que requer trabalho coletivo, mas que lhe são atribuídos grande parte da responsabilidade para lidar diretamente com a comunidade, Que exige habilidades e compreensão do uso de instrumentos e tecnologias complexas e inovadoras, por vezes desconhecidas e ausentes no processo de treinamento/ capacitação ${ }^{(1,8)}$.
É evidente a necessidade de reforçar a proposta do trabalho em equipe no PSF, devido à complexidade e dinamicidade das necessidades biológicas, psicológicas e sociais das famílias. O PSF pode se abrir para um trabalho em equipe com um projeto comum, onde os trabalhos de cada profissional seja autônomo, criativo e complementar ao trabalho do outro e o foco central seja a família e seu entorno ${ }^{(21)}$. As autoras enfatizaram Que para esse novo processo de trabalho deve existir a multiprofissionalidade Que é a atuação conjunta de vários profissionais e mais ainda, a interdisciplinaridade, Que é a produção do conhecimento a partir da integração de várias disciplinas e áreas do conhecimento. Destacaram a necessidade de formação e capacitação de profissionais de saúde Que tenham competência para esse complexo e contínuo trabalho de mudança.

O trabalho em equipe multidisciplinar com atuação interdisciplinar, está pautado nos seguintes critérios: comunicação entre os agentes de trabalho, articulação das ações, reconhecimento das diferenças técnicas entre os trabalhos especializados, o Questionamento das desigualdades estabelecidas entre os diversos trabalhadores e o reconhecimento do caráter interdependente da autonomia profissional ${ }^{(22)}$. Quando a equipe direciona seu trabalho nessa perspectiva estabelece o que a autora chamou de "equipe integrada" e tende a construir um projeto comum e articulado, distanciando-se do que a autora definiu como "equipe agrupamento" Que equivale a simples existência de vários trabalhadores no mesmo espaço laboral.

\section{DSC 2- Percepção do ACS sobre as práticas desenvolvidas pelo PSF:}

Aqui a gente desenvolve o trabalho com pré-natal, crescimento e desenvolvimento, grupos de hipertensos e diabéticos, informações sobre hantavirose, dengue essas doenças que tem sido febre agora. Nós fazemos também o trabalho de esclarecimento sobre doenças sexualmente transmissíveis, grupos familiares e essas coisas. A principal atividade que o ACS faz é a visita domiciliar. Também fazemos palestras na escola, na comunidade e nos grupos de hipertensos e diabéticos da unidade. No Colégio São Paulo, na época da hantavirose, foi cerca de pelo menos duas semanas de palestras, falando só sobre isso e teve a dengue também. O PSF é muito importante pra comunidade porQue é um trabalho preventivo. Ninguém Quer se prevenir espera acontecer e ai vem até o PFS e Quer ser atendido. $O$ que foi passado no curso é Que o PFS serviria como uma medida preventiva e não esta servindo dessa maneira. Esta funcionando mais como uma emergência. Eles vêm pra cá machucados e aqui não tem como a gente fazer muita coisa. $O$ pessoal se preocupa muito com o médico e remédio. Até porQue teve uma época Que a gente não tinha médico e aí o pessoal falava: ah mais o Que vocês estão fazendo na área Que não tem médico (DSC2).

O DSC2 demonstra Que são desenvolvidas ações básicas a grupos específicos e vulneráveis como: diabéticos, crianças, gestantes hipertensos, palestra para prevenção de doenças e promoção da saúde. Todavia deve ser destacado Que as atividades são realizadas como momentos isolados no processo de trabalho e desvinculados de um modelo de programação em saúde voltado para crianças, adolescentes, mulheres, idosos, Que tome como base para organização das ações o perfil epidemiológico da comunidade segundo(19).

No Que tange as ações de prevenção e promoção da saúde, sobretudo, as ações educativas junto à comunidade sabe-se Que 
são potentes instrumentos de trabalho intramuro e extramuro, mas foi observado Que reQuer maior aprofundamento e utilização de novas tecnologias, pois os conteúdos baseiam-se no enfoque higienista Que preconizam intervenções normalizadoras ${ }^{(1-14)}$.

As visitas domiciliares realizadas pelo ACS podem ser profícuos espaços dialógicos de trabalho ${ }^{(15)}$, pois os moradores solicitam ajuda para si ou para o vizinho, para a resolução de problemas de saúde, saneamento, meio ambiente, entre outros.

O discurso coletivo releva Que é imprescindível o uso de tecnologias educacionais Que motivem as pessoas à prevenção e promoção da saúde, porQue se percebe a ausência de comportamentos pessoais frente ao autocuidado, Que são motivados por fatores histórico, social e educacional. Nesse sentido, a da cultura de medicalização Que está impregnada, é empecilho para mudar o modo de cuidar da saúde.

Em suma, os dados sobre as práticas desenvolvidas mostram Que contêm ações Que tem sido denominado de ingredientes da cesta básica de serviços ofertados pelo PSF, Que se caracteriza como medicina simplificada, Que usa tecnologia de baixo custo voltada para população carente de recursos de saúde e de infraestrutura urbana ${ }^{(11,23)}$.

\section{DSC 3- Percepção do ACS sobre a finalidade do: acesso aos serviços de saúde}

O PSF descongestionou os Postos de Saúde (PS), Centro de Saúde (CS), Pronto Socorro e os Hospitais. Porque geralmente no Posto de Saúde a fila é enorme. Os Hospitais estão sempre superlotados, a emergência daqui, pra adulto, só funciona a partir da noite. Só de saber que os Postos de Saúde hoje em dia não estão mais congestionados como eram antes, sobrecarregados. Antigamente? Nossa, era um transtorno! Agora não. Tem casos Que se resolve aQui mesmo. Hoje em dia está mais controlado. Quando vamos ao Centro de Saúde vemos Que o movimento está sempre assim, controlado. As pessoas já vêm aQui, procuram mais aQui e não vão mais ao Centro de Saúde. Mesmo os Que moram lá perto vêm pra cá porQue lá é muito cheio. Então hoje a gente tem acesso a médico, enfermeiro, coisas Que há tempos atrás a gente não tinha. Tem um livre acesso: trazer a comunidade ao médico e levar o médico em casa, nas famílias. Uma dificuldade é Quando chega alguém machucado aQui a gente manda pro PS e aí chega lá no PS, eles mandam pra gente e fica aQuela guerra e fica muito ruim trabalhar $\operatorname{assim}(D S C 3)$

O DSC3 revela que uma das finalidades do PSF é facilitar o acesso aos serviços de saúde, diminuir filas e descongestionar os demais serviços. O acesso ou acessibilidade ${ }^{(24)}$, indica o grau de facilidade com que a pessoas obtêm o cuidado. Ressalta-se Que Quanto às formas de avaliação do acesso há diferentes propostas, ou seja, a avaliação do acesso feita em relação aos resultados ou objetivos finais dos sistemas de saúde ou na relação entre vários elementos Que compõem o sistema para atingir esses objetivos. As autoras discutiram também o conceito de "uso dos serviços"(24) como dependente do comportamento individual de Quem o procura e dos profissionais Que fazem o atendimento. São considerados como determinantes do uso de serviços os fatores relacionados: às necessidades de saúde, aos usuários, aos prestadores de serviços, à organização do serviço, à política de saúde. Assim, para estas autoras, o acesso, apesar das divergências relaciona-se a características da oferta dos serviços e o uso depende de fatores individuais e contextuais, embora se observe uma tendência de deslocamento do seu eixo da entrada nos serviços (uso) para os resultados dos cuidados recebidos. Concluíram que os indicadores de uso impactam diretamente a doença, mas apenas indiretamente a saúde ${ }^{(24)}$.

Verifica-se, portanto, Que embora seja positiva a avaliação do coletivo de que o acesso é fácil em decorrência da unidade ser próxima da residência do usuário esse não é o único critério para se analisar a acessibilidade, mas pode ser considerado o mais importante, pois ao primeiro sinal de desconforto a população vai ao PSF.

O requisito primordial para Que a unidade PSF seja porta de entrada do sistema de saúde é Que seja acessível à população adstrita, eliminando-se barreiras financeiras, geográficas, temporais, administrativas e culturais e Que deve constituir-se em serviço de procura regular e servir de filtro e ponto de entrada do fluxo pela rede assistencial buscada pelos usuários ${ }^{(25)}$.

Nessa direção, os dados como dificuldades relacionadas à estratégia: a comunicação com outros serviços, a necessidade do PSF assumir a coordenação da assistência à população Que reQuerem diferentes ações, considerando-se as fases do ciclo de vida, dos diversos níveis de complexidade assistencial.

A avaliação nacional da integração do PSF à rede básica de serviços de saúde realizada em dez grandes centros urbanos ${ }^{(25)}$, confirmaram o mesmo problema: um terço ou mais dos profissionais de nível superior das eQuipes de saúde da família informaram nunca receber Qual Quer tipo de contra-referência. As centrais de marcação de consulta não tem sido suficientes para solucionar esse problema, portanto, o grande desafio é melhorar a comunicação entre profissionais e serviços. Esta mesma pesquisa demonstrou ainda Que em algumas cidades pesQuisadas havia um meio de comunicação sistemática entre serviços mediada pela gerência territorial com definição de especialistas e/ou profissionais de referência para as unidades básicas de cada território ${ }^{(25)}$.

\section{DCS 4 - Percepção do ACS sobre necessidades de continuidade e dificuldade do PSF}

Era muito formal o convívio com a comunidade e a saúde. Hoje é mais familiarizado, comum e fácil. Mas falta material pra trabalhar, falta papel, caneta, falta de tudo e também falta treinamento. Só o Que falta pra completar o nosso PSF aQui é dentista. A importância de trabalhar no PSF é Que eu estou servindo à comunidade, prestando um serviço para a comunidade e para a sociedade em si. A comunidade adora o PSF. Gostam muito mesmo. É um programa de grande valor pra comunidade. É muito positivo. É dez. No início estava meio devagar, mas agora esta funcionando legal, está andando. Esta cada vez melhor. Era muito formal o convívio de comunidade e saúde, mas hoje é mais familiarizado. Eu gostaria que o programa fosse fixo como foi prometido pelo governo. PorQue o programa: vêm Quatro anos..., e é mais política. A comunidade acredita, a gente desempenha o máximo, monta um serviço e Quando está tudo aQuilo montado, ai cai tudo e começa tudo de novo. A agente que nem estava no programa anterior escuta: será Que esse programa vai ficar? Será Que se eu sair do CS pra continuar um pré-natal ou um CD com vocês... Vocês não vão sair? Por isso eu acho que o PFS deve permanecer fixo igual a um centro de saúde e não deve sair para não deixar a comunidade descrente (DSC 4).

A proximidade, informalidade e a familiaridade são expressões do sujeito coletivo sobre o PSF, revelando importantes elementos como o estabelecimento do vínculo e da escuta na relação entre a 
equipe e as famílias adscritas ${ }^{(15)}$. Foi observado no processo de trabalho da unidade Que, embora haja um esforço individual em relação ao acolhimento, não dispõe de instrumentos suficientes para fortalecer a potencialidade dessa tecnologia e para desencadear uma ação mais resolutiva à demanda identificada ${ }^{(1)}$.

O DSC4 demonstra Que há falta de meios e instrumentos (materiais e não materiais), o que corrobora com o observado na coleta de dados: como a falta de materiais para as ações educativas, desde o espaço físico inadequado até a Quantidade insuficiente de cadeiras para a atividade grupal, instrumentos para operacionalizar o curativo, medicação, profissionais, dentre outros.

Evidencia-se Que a comunidade tem percepção bastante positiva do programa, principalmente porQue permitiu maior aproximação entre a população o serviço de saúde. Entretanto, a descontinuidade políticoadministrativa causa insegurança e descrença no PSF segundo a percepção do ACS. As rupturas ocorridas durante a implantação do programa no DF interferiram negativamente no processo de trabalho, pois as equipes sofreram com a rotatividade de pessoal e o envolvimento construído com a comunidade era rompido.

Esta descontinuidade está pautada na mudança de governo e na rotatividade dos agentes não só da equipe de saúde da família, como da supervisão e coordenação central do programa. A descontinuidade política é identificada após os pleitos eleitorais Quando há extinção da proposta vigente e nascimento de nova proposta, o Que é um equívoco político pela natureza irreversível da consolidação da estratégia nacional, porém, desestabiliza o processo de trabalho e as relações entre famílias-equipe PSF. Houve um apelo à fixação do PSF como um CS para garantir Qualidade, vínculo, crédito da população e permanência do programa o Que fortalece o sentimento de pertencimento do ACS e seu desejo de vivenciar a estratégia para a consolidação do SUS e refutá-la eneuanto um programa paralelo.

\section{CONSIDERAÇÕES FINAIS}

Os resultados demonstram Que na percepção dos ACS o objeto de trabalho do PSF é a comunidade com seus problemas de saúde, Que devido a sua complexidade reQuer trabalho coletivo. Em relação às praticas desenvolvidas revela-se Que são desenvolvidas ações básicas que tem a finalidade de facilitar o acesso da população aos serviços de saúde, principalmente diminuir as filas e o congestionamento da demanda Que recorre às demais unidades do sistema de saúde, é um trabalho preventivo convivendo com a busca por atendimento curativo e de emergência. Na percepção dos ACS A população tem uma compreensão eQuivocada sobre a finalidade do processo de trabalho em saúde do PSF, manifestando seu interesse pela consulta médica e pelo remédio.

Para Que o PSF torne-se uma porta de entrada do sistema de saúde pública facilitando o acesso da população a outros níveis de assistência e transforme, de fato, as relações interpessoais do atendimento as famílias estabelecendo vínculo, humanização e acolhimento, com compromisso ético-político de resolutividade e integralidade, fazem-se necessário tornar público os novos paradigmas da saúde e da organização/funcionamento da rede de saúde pública para a população, garantir instrumentos e meios para atenção básica de Qualidade, reconstruir o conteúdo das práticas de forma a garantir a atenção aos problemas das famílias, estabelecer o acolhimento em todos os momentos do processo de trabalho e por todos os profissionais, utilizar a clínica centrada no usuário com escuta e comprometimento ético, avançar na implementação de intervenções intersetoriais organizadas sob a concepção da Vigilância à Saúde, Promoção da Saúde e Cidades Saudáveis.

A saúde da família como estratégia tem alcançado seu objetivo de alterar a hegemonia do modelo de atenção atual, principalmente na esfera da atenção básica ofertada para a população do seu território de referência. Este é um grande passo, entretanto, ainda precisa se consolidar técnica e politicamente para promover mudanças nas práticas de saúde da rede de serviços como um todo.

Por fim, deve se destacar Que, enQuanto contingente de força de trabalho o ACS representa uma cifra importante no cenário dos trabalhadores da saúde, o Que não é suficiente, mas necessário para mudar a correlação de forças entre o velho e o novo modelo de atenção à saúde, desde Que eles disponham de elementos para a transformação do seu processo de trabalho num diálogo permanente como outros setores da sociedade e a participação de gestores, equipes técnicas e população.

\section{REFERÊNCIAS}

1. Silva IA, Dalmaso ASW. Agente comunitário de saúde: o ser, o saber, o fazer. Rio de Janeiro (RJ): Fiocruz; 2002.

2. Minayo MCS. Programa Agente de Saúde do Ceará: estudo de caso. Fortaleza (CE): UNICEF; 1990.

3. Sousa MF. Agentes comunitários de saúde: choque do povo. São Paulo (SP): Hucitec; 2002.

4. Ministério da Saúde (BR). Programa Saúde da Família: saúde dentro de casa. Fundação Nacional de Saúde/Departamento de Operações/Coordenação de Saúde da Comunidade. Brasília (DF): Ministério da Saúde; 1994

5. Ministério da Saúde (BR). Saúde da Família: uma estratégia para a orientação do modelo assistencial. Secretaria de Assistência à Saúde/Coordenação de Saúde da Comunidade. Brasil (DF): Ministério da Saúde; 1997.

6. Ministério da Saúde (BR). Agentes Comunitários de Saúde, Equipe Saúde da Família e Equipes de Saúde Bucal em atuação 2005. [citado em 2007 abr 23]. Disponível em: URL: http// portal saúde.gov.br/saúde.

7. Nunes OM, Trad BL, Almeida BA, Homem CR, Melo MCI. O agente comunitário de saúde: a construção da identidade híbrida e polifônica. Cad Saúde Pública 2002; 18(6): 1639 46.

8. Nogueira PN, Ramos SBF, Vale VO. A vinculação institucional de um trabalhador "sui generis": o Agente Comunitário de Saúde. Cadernos IPEA 2002.

9. Paim IS. Vigilâncias da Saúde: dos modelos assistenciais para a promoção da saúde. In: Czeresnia D, organizador. Promoção da Saúde: conceitos, reflexões, tendências. Rio de Janeiro (RJ): Fiocruz; 2003. p. 161-74.

10. Teixeira CF. A mudança do modelo de atenção à saúde no SUS: desatando nós, criando laços. Saúde Debate 2003; 27(65): 257-77.

11. Merhy EE. A rede básica como uma construção da saúde pública e seus dilemas. In: Merhy EE, Onocko R, organizadores. Agir em Saúde: um desafio para o público. $2^{\mathrm{a}}$ ed. São Paulo (SP): Hucitec; 2006. p. 197-228.

12. Teixeira CF. Saúde da Família, promoção e vigilância: construindo a integralidade da atenção à saúde no SUS. In: 
Teixeira CF, Solla JP. Modelo de Atenção à Saúde: promoção, vigilância e saúde da família. Salvador (BA): EDUFBA; 2006. p:59-83.

13. Paim IS. O objeto e a prática da saúde coletiva: o campo demanda um novo profissional? In: Paim IS. Desafios para a Saúde Coletiva no Século XXI. Salvador (BA): EDUFBA, 2006. p. 99-116.

14. Mishima SM, Pereira MJB, Fortuna CM e Matumoto S. Trabalhadores de saúde: problema ou possibilidade de reformulação do trabalho em saúde? In: Falcão A, organizadores. Observatório de Recursos Humanos em Saúde. Rio de Janeiro (RI): Fiocruz; 2003. p. 137-56

15. Schimith MD, Lima MADS. Acolhimento e vínculo em uma equipe do PSF. Cad Saúde Pública 2004; 20(6): 1487-94.

16. Lefévre F, Lefévre AMC. O Discurso do Sujeito Coletivo: um enfoque em pesquisa Qualitativa (desdobramentos). Caxias do Sul (RS): EDUCS; 2003.

17. Lefévre F, Lefévre AMC. Depoimentos e Discursos: uma proposta de análise em perspectiva social. Brasília (DF): Líber Livros Editora; 2005.

18. Donnângelo MCF, Pereira L. Saúde e sociedade. São Paulo
(SP): Duas Cidades; 1976

19. Schraiber LB, Nemes MIB, Mendes-Gonçalves RB, organizadores. Saúde do adulto: programas e ações na unidade básica. $2^{\text {a }}$ ed. São Paulo (SP): HUCITEC; 2000. p. 29-47.

20. Bartolomei CEF, Carvalho MS, Deldueue MC. A saúde é direito! Saúde em Debate 2003; 27(65): 184-9.

21. Almeida MCP, Mishima SM. O desafio do trabalho em equipe na atenção à saúde da família: construindo novas autonomias no trabalho. Interface Comunic Saúde Educ 200 I ; 9: 150-5.

22. Peduzzi M. Equipe multiprofissional de saúde: conceito e tipologia. Rev Saúde Pública 200 I; 35(1): 103-9.

23. Aleixo JLM. A atenção primária à saúde e o programa de saúde da família: perspectivas de desenvolvimento no início do terceiro milênio. Rev Mineira Saúde Pública 2002; I (1): I-I6.

24. Travassos C, Martins M. Uma revisão sobre o conceito de acesso e utilização de serviços de saúde. Cad Saúde Pública 2004; 20(2): 190-8.

25. Giovanella L, Escorel S, Mendonça MH. Porta de entrada pela atenção básica Integração do PSF à rede de serviços de saúde. Saúde em Debate 2003; 27(65): 278-89. 